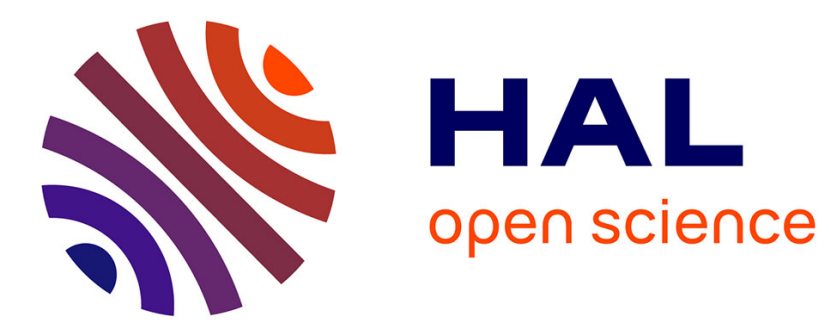

\title{
Is low frequency excess noise of GMI induced by magnetization fluctuations?
}

\author{
Christophe Dolabdjian, Basile Dufay, Sébastien Saez, Arthur Yelon, David \\ Ménard
}

\section{- To cite this version:}

Christophe Dolabdjian, Basile Dufay, Sébastien Saez, Arthur Yelon, David Ménard. Is low frequency excess noise of GMI induced by magnetization fluctuations?. International Conference on Materials and Applications for Sensors and Transducers (ICMAST), 2013, prague, Czech Republic. hal00988089

\section{HAL Id: hal-00988089 \\ https://hal.science/hal-00988089}

Submitted on 7 May 2014

HAL is a multi-disciplinary open access archive for the deposit and dissemination of scientific research documents, whether they are published or not. The documents may come from teaching and research institutions in France or abroad, or from public or private research centers.
L'archive ouverte pluridisciplinaire HAL, est destinée au dépôt et à la diffusion de documents scientifiques de niveau recherche, publiés ou non, émanant des établissements d'enseignement et de recherche français ou étrangers, des laboratoires publics ou privés. 


\title{
Is low frequency excess noise of GMI induced by magnetization fluctuations?
}

\author{
DOLABDJIAN Christophe ${ }^{1, a}$, DUFAY Basile ${ }^{1, b}$, SAEZ Sebastien ${ }^{1, c}$, \\ YELON Arthur ${ }^{2, \mathrm{~d}}$ and MENARD David ${ }^{2, \mathrm{e}}$
}

${ }^{1}$ Normandie Univ, France ; UCBN, GREYC, F-14032 Caen, France; CNRS, UMR 6072, F-14032 Caen, France

${ }^{2}$ Ecole Polytechnique de Montréal, Département de Génie Physique \& Regroupement Québécois des Matériaux de Pointe, Montréal, Québec, Canada H3C3A7

achristophe.dolabdjian@unicaen.fr, bdufay.basile@unicaen.fr, 'sebastien.saez@unicaen.fr, darthur.yelon@polymtl.ca, 'david.menard@polymtl.ca

Keywords: GMI, Noise, Magnetization fluctuations, Magnetometers.

\begin{abstract}
We have investigated the possible impact of low frequency magnetization fluctuations on the equivalent magnetic excess noise of GMI sensors, which we have recently shown to exhibit $1 / f$ noise. This noise component is not associated with the detailed measuring setup nor with the conditioning electronic noise sources, suggesting that it is intrinsic to the sensing element. Various intrinsic GMI noise sources might be able to explain this observation; these include magnetic domain wall motion, hysteresis loop losses, etc. Since GMI elements are excited by a high frequency current, it has been assumed that these low frequency (lf) intrinsic noise sources cannot interact with the carrier and the sensed signal. We recall that the GMI effect is based on an impedance variation, which is governed by magnetization angle variations. These are modulated by the sensed signal and the lf magnetization noise, which then appear as sidebands around the carrier frequency. Applying the fluctuation-dissipation theorem to the GMI model, we have related both the signal and noise to the magnetic susceptibility spectrum and thus quantified the equivalent magnetic noise of GMI sensors at lf. We then present a preliminary comparison to our previous experimental results.
\end{abstract}

\section{Introduction}

Giant MagnetoImpedance (GMI) in soft magnetic materials has considerable potential for magnetic field sensing applications [1,2], for which high sensitivity to the magnetic field and low equivalent magnetic noise spectral density are required. In the past few years, a theoretical model of GMI sensor noise, treating its dependence upon material properties has been developed, [3,4,]. It has been applied to the white noise regime [5], for which we found that electronic noise dominates and explains the discrepancies between the expected, intrinsic, GMI noise and experimental noise measurement. In contrast, the If, $1 / f$, noise, which has been observed, has not been theoretically investigated. This work focuses on this issue. A first theoretical estimate of the intrinsic magnetic noise at If is proposed, in order to evaluate the fundamental limitations of GMI sensing elements. Expected wire sensor noise performances are reported and compared with experimental observations.

\section{Noise in GMI sensing elements}

It has been recognized that thermal fluctuations of the magnetization set fundamental limits to the signal-to-noise ratio of magnetoresistive sensors [6]. The response of any magnetic field sensor, which depends upon the magnetization direction of its sensing elements, is subject to intrinsic magnetic noise arising from thermal fluctuations of the magnetization. For a soft magnetic wire, 
driven by a high frequency longitudinal current, such as a GMI sensing element, we have presented a model, discussed above, with which we may evaluate intrinsic GMI sensor white noise [3,4]. Here, we extend it to include the fluctuation-dissipation theorem for the magnetization at lf. Based on a small signal analysis, the voltage appearing across the GMI is usually given by

$$
V(t)=\left(Z+\frac{\partial Z}{\partial B} \cdot b(t)+z_{n}(t)\right) I(t)
$$

where $Z$ is the impedance of the wire at the working magnetic field point, and $\partial Z / \partial B$ is the impedance sensitivity; with $B$ the magnetic induction; $b(t)$ is the small sensed applied magnetic induction, $z_{n}(t)$, the equivalent impedance noise variation and $I(t)$ the sinusoidal rf current of amplitude and angular frequency, $I_{A C}$ and $\omega_{0}$.

By using classical demodulation technique and considering chain gains to be unity, the Fourier Transform of the output signal of the lock-in is

$$
V(\omega)=\frac{I_{A C}}{2}\left(Z\left(\omega_{0}\right)+\frac{\partial\left(Z\left(\omega_{0}\right)\right)}{\partial B} \cdot b(\omega)+z_{n}(\omega)\right),
$$

where $\omega(=2 \pi f)$ is the angular frequency. At the working angular frequency, $\omega_{0}$, it yields sensor field sensitivity, in $\mathrm{V} / \mathrm{T}$, of

$$
S=\frac{I_{A C}}{2} \frac{\partial Z\left(\omega_{0}\right)}{\partial B}
$$

and an equivalent voltage noise, in $\mathrm{V} / \sqrt{\mathrm{Hz}}$, of

$$
v_{n}(\omega)=\left(\frac{I_{A C}}{2}\right) z_{n}(\omega) .
$$

We assume that the cylindrical shell volume of the GMI wire, defined by the skin depth, is a single domain with a uniform helical magnetization, subject to a uniform effective internal field dominated by magnetoelastic anisotropy. Then, the impedance of the wire, $Z$, obtained from the solution of Maxwell's equations and the linearized Landau-Lifshitz equation for magnetization dynamics, can be approximated by $[3,4]$

$$
Z=Z_{0}+\Delta Z \cos ^{2} \theta
$$

where $\theta$ is the angle between the shell layer average magnetization and the wire longitudinal axis (see Fig. 1 of [3]). In Eq. (5), $\Delta Z=\left(Z_{M}-Z_{0}\right)$, where $Z_{0}$ and $\left(Z_{M}=\sqrt{\mu_{t}} Z_{0}\right)$ are the non-magnetic and magnetic parts of the wire impedance, corresponding to small circumferential driving fields which are parallel and perpendicular to the static magnetization, respectively, and $\mu_{t}$ is the relative transverse permeability.

We assume that the driving field, $h_{\varphi}$, static applied field, $H_{0}$, and effective anisotropy field, $H_{K}$, are all small compared to the saturation magnetization, $M_{\mathrm{s}}$.as is usually the case for GMI. We further assume that the impedance noise power spectral density, $S_{h h}$, depends upon the thermal fluctuations of the free layer magnetization. Assuming equipartition theorem and our previous model, as was done in refs. $[3,4]$, we find 


$$
\overline{z_{n}^{2}(\omega)} \approx\left(\frac{\partial Z}{\partial \theta}\right)^{2} S_{\theta \theta}(\omega) \approx\left(\frac{\partial Z}{\partial \theta}\right)^{2} \frac{\left.\chi_{t}^{2}(\omega)\right|_{\omega \rightarrow 0} S_{h h}}{M_{s}^{2}} \approx\left(\frac{\partial Z}{\partial \theta}\right)^{2}\left(\frac{1}{M_{s}}\right)^{2}\left(\frac{4 k_{B} T \chi^{\prime \prime}}{\vartheta \omega \mu_{0}}\right)
$$

In Eq. (6) $S_{\theta \theta}$ is the spectral density of the magnetization direction fluctuations, $\chi_{t}$, the magnetic susceptibility, $\vartheta$, the free layer wire volume, $\mu_{0}$ the permeability of free space, and $k_{B} T$, the thermal energy. The imaginary part of the susceptibility $\chi^{\prime \prime}$ is usually thought of as the coefficient that describes the losses driven by applied fields.

After some simplification, based on our previous description [3], the equivalent impedance noise spectral density may be rewritten as

$$
\overline{z_{n}^{2}(\omega)} \approx\left(\frac{\partial Z}{\partial \theta}\right)^{2}\left(\frac{4 k_{B} T}{\vartheta \omega \mu_{0} M_{s}^{2}} \tan (\delta)\right) \approx\left(\frac{\partial Z}{\partial \theta}\right)^{2}\left(\frac{4 k_{B} T}{\vartheta \omega \mu_{0} H_{i n t}^{2}} \tan (\delta)\right)=\frac{c}{\omega} \alpha \frac{1}{f},
$$

where $c$ is a constant and $\tan (\delta)\left(=\frac{\chi^{\prime \prime}}{\chi^{\prime}}\right)$ is the magnetic loss tangent factor (assuming $\chi^{\prime} \approx 1$ for $\omega \rightarrow 0$ ). Let us assume [4], a wire with circumferential anisotropy such that the magnetization versus field relation is given by $\cos \theta=H_{0} / H_{K}$, and thus the internal field is $H_{\text {int }}=\left(H_{K}^{2}-H_{0}^{2}\right) / H_{K}$. The sensor will be operated at a few $\mathrm{MHz}$ with a DC bias field equal to $H_{0}=H_{K} / 2$. In these conditions, the transverse permeability is well approximated by $\mu_{t}=M_{s} / H_{\mathrm{int}}$. We notice that

$$
\frac{\partial Z}{\partial \theta} \approx Z_{0} \sqrt{\frac{3 \mu_{t}}{4}}
$$

Also, based on these hypotheses, Eqs. (7) and (8) lead to a voltage noise power spectral density, in $\mathrm{V}^{2} / \mathrm{Hz}$, given by

$$
\overline{v_{n}^{2}(\omega)} \approx \frac{I_{0}^{2}}{4}\left(Z \sqrt{\frac{3 \mu_{t}}{4}}\right)^{2} \frac{4 k_{B} T}{\vartheta \omega \mu_{0} H_{i n t}^{2}} \operatorname{Tan}(\delta) \approx \frac{I_{0}^{2} Z^{2}}{2}\left(\frac{4 k_{B} T \alpha}{\vartheta \gamma \mu_{0}^{2} H_{K}^{3}}\right) \frac{\operatorname{Tan}(\delta)}{\alpha \frac{f}{f_{m}}} .
$$

In Eq. (9) $\gamma$ is the gyromagnetic ratio, $\alpha$, the dimensionless Gilbert damping parameter and $f_{m}$ the ferromagnetic resonance frequency of the wire. Then, the equivalent magnetic noise spectral density, in $\mathrm{T} / \sqrt{\mathrm{Hz}}$, in the lf, $1 / f$, regime is given by

$$
\overline{b_{n}^{2}(f)^{1 / 2}} \approx \frac{Z \sqrt{2}}{\left|\frac{\partial Z\left(f_{0}\right)}{\partial B}\right|} \sqrt{\frac{4 k_{B} T f_{m} \operatorname{Tan}(\delta)}{\vartheta \gamma \mu_{0}^{2} H_{K}^{3} f}} .
$$

Equation (10) is the main result of this section. It shows that the equivalent magnetic noise spectral density is expected to scale directly with the impedance sensitivity ratio, with the square root of the ferromagnetic resonance frequency, the magnetic loss tangent coefficient, and the absolute temperature, and inversely with the square root of the wire volume, the gyromagnetic ratio and the anisotropy field. 


\section{Results and discussion}

Eq. (10) may be compared to the equivalent magnetic spectral density in the white noise regime, obtained earlier 3,[4]:

$$
\overline{b_{n_{-} w}^{2}(\omega)} \approx \frac{Z^{2}}{\left|\frac{\partial Z(\omega)}{\partial B}\right|^{2}}\left(\frac{4 k_{B} T \alpha}{\vartheta \gamma \mu_{0}^{2} H_{K}^{3}}\right) .
$$

so that

$$
\frac{\overline{b_{n}^{2}(f)}}{\overline{b_{n_{-}}^{2}(f)}}=2 \frac{f_{m}}{f} \frac{\operatorname{Tan}(\delta)}{\alpha} .
$$

Commercial melt-extracted magnetic amorphous wires (MXT, Inc. of Montreal) with a composition of $\mathrm{Co}_{80.89} \mathrm{Fe}_{4.38} \mathrm{Si}_{8.69} \mathrm{~B}_{1.52} \mathrm{Nb}_{4.52}$ (wt\%), were used as reference sensing elements. The samples considered were two centimeter long of approximately $30 \mu \mathrm{m}$ in diameter. An average resistivity of $130 \mu \Omega \mathrm{cm}$ was inferred from the resistance of the wires. This yields an impedance value of around $50 \Omega$ in the $\mathrm{MHz}$ frequency range with a highest sensitivity close to $100 \mathrm{M} \Omega / \mathrm{T}$. The magnetic noise density, calculated from Eq. (11) with $\alpha=0.01, \gamma=28 \mathrm{GHz} / \mathrm{T}$ and $H_{K}=55 \mathrm{~A} / \mathrm{m}$, is about $10 \mathrm{fT} / \sqrt{\mathrm{Hz}}$. Assuming $f_{m} \approx \gamma \mu_{0} M_{S}$ and a loss factor of 0.001 to 0.01 , the rhs of Eq. (12) is about $\left((4-40) 10^{6}\right) / f$. So, it yields to an equivalent magnetic noise spectral density of between 27 and $86 \mathrm{pT} / \sqrt{\mathrm{Hz}}$ at $1 \mathrm{~Hz}$ and having a $1 / f$ noise behavior. This theoretical value maybe compared to our previous experimental results [5, 7], which give noise levels of 30-100 pT/Hz at $1 \mathrm{~Hz}$.

The result, Eq. (10) must be viewed only as a rough estimate of the equivalent GMI magnetic noise at lf. These preliminary calculations suggest that thermal magnetic noise arising from thermal fluctuations of the magnetization could be a significant contribution to the if intrinsic noise of the sensing element through impedance fluctuations. Further theoretical and experimental studies are underway to clarify this issue and to fix limits of the lf noise performance of GMI, a key point for the future of GMI sensor development.

\section{References}

[1] S. Sandacci, D. Makhnovskiy, L. Panina, K. Mohri, and Y. Honkura: Magnetics, IEEE Transactions on, 40(6), 3505-3511(2004)

[2] Manh-Huong Phan, Hua-Xin Peng: Materials Science 53, 323-420(2008)

[3] D.Ménard, G.Rudkowska, L.Clime, P.Ciureanu, S.Saez, C.Dolabdjian, D.Robbes, A.Yelon: Sensors and Actuators A: Physical - 129(1-2), 6-9(2006)

[4] L.Melo, D.Menard, A.Yelon, L.Ding, S.Saez, C.Dolabdjian: Journal of Applied Physics, 103(3), 033903 1-6(2008)

[5] B.Dufay, S.Saez, C.Dolabdjian, A.Yelon, D.Menard: IEEE Sensors Journal, 13(1), 379388(2013)

[6] W.F.Egelhoff Jr., P.W.T.Pong, J.Unguris, R.D.McMichael, E.R.Nowak, A.S.Edelstein, J.E.Burnette, G.A.Fischerd: Sensors and Actuators A: Physical - 155(2), 217-225(2009)

[7] L.Ding, S.Saez, C.Dolabdjian, L.Melo, D.Menard, A.Yelon: IEEE Sensors Journal, 9(2), 159168(2009) 